\title{
NARROWING OF SPECTRAL LINES BEYOND THE NATURAL OR DEPHASING LINE WIDTH
}

\author{
W. ZINTH, M.C. NUSS and W. KAISER \\ Physik Department der Technischen Universität München, \\ München, Fed. Rep. Germany
}

Received 11 October 1982

\begin{abstract}
Transient excitation and gated or delayed observation provides a narrowing of the inherent line wid th of the transition. Possible experimental one- and two-photon systems are discussed and the loss of signal with line narrowing is calculated. The general case of lines broadened by dephasing processes is treated. Contact is made with the line narrowing in recent Raman type experiments.
\end{abstract}

\section{Introduction}

In spectroscopy one is always faced with the problem of spectral resolution. One wishes to determine the frequency of the quantized transition with highest accuracy and one is interested in detecting fine structure or neighboring transitions. Disregarding experimental factors, the ultimate resolution is dictated by the inherent linewidth of the transitions. In optical spectroscopy we know various line-bruadening processes. We point to the Doppler effect or to collision broadening in gases, to dephasing processes in condensed phases and to the population lifetime which gives rise to the natural linewidth.

In recent years different novel techniques have been devised which provide spectral resolution beyond the transition linewidth. For instance, Doppler broadening can be eliminated by saturation spectroscopy or by two counter-propagating beams for two-photon transitions [1]. Even measurements beyond the natural line width have been performed taking biased signals from the fluorescent decay [25]. Techniques have been proposed where the difference between the decay rates of the two states rather than their sum determines the linewidth $[6,7]$, and narrowing of the natural linewidth by decaying-pulse excitation has been discussed [8].

Very recently we have demonstrated substantial line narrowing of Raman type transitions in con- densed phases $[9,10]$. The lines were broadened by vibrational dephasing. It is the aim of this article to show new possibilities of line narrowing in coherent transient experiments. In our treatment we include dephasing processes. Without dephasing our results are applicable to line narrowing beyond the natural linewidth.

\section{Theory.}

We note that the spectral resolution in a transient experiment is not limited by the lifetime of the investigated levels, but is determined by the specific experiment. Under favorable conditions the observed line may become substantially narrower than the spontaneous width measured in a steady-state experiment. We consider an ensemble of two-level systems with ground state $\langle b\rangle$ and excited state $|a\rangle$ at the fre. quency difference $\omega_{0}$. The interacting driving force at the frequency $\nu$ is denoted by $\widetilde{A}(t)$. For the elements of the corresponding density matrix $\rho$ we obtain the equations:

$$
\begin{aligned}
& \dot{\rho}_{a a}=-\gamma_{a} \rho_{a a}-\mathrm{i} \widetilde{A}(t)\left(\rho_{b a}-\rho_{a b}\right), \\
& \dot{\rho}_{b b}=-\gamma_{b} \rho_{b b}+\mathrm{i} \widetilde{A}(t)\left(\rho_{b a}-\rho_{a b}\right), \\
& \dot{\rho}_{a b}=-\left(\mathrm{i} \omega_{0}+\gamma_{a b}\right) \rho_{a b}-\mathrm{i} \widetilde{A}(t)\left(\rho_{b b}-\rho_{a a}\right) .
\end{aligned}
$$


In the following discussion the driving force $\widetilde{A}(t)$ is caused by electro-magnetic fields. For one-photon or two-photon processes $\widetilde{A}(t)$ is proportional to $E(t)$ or $E_{i}(t) E_{j}(t)$, respectively. The diagonal elements $\rho_{a a}$ and $\rho_{b b}$ describe the population of the two levels with damping constants $\gamma_{a}=1 / T_{1}$ and $\gamma_{b}$, respectively. Here we only treat the case of small excitation where $\rho_{b b} \approx 1 \gg \rho_{a a}$. The off-diagonal elements $\rho_{a b}=\rho_{b a}^{*}$ represent the coherent excitation of the system. $\rho_{a b}$ relaxes with the dephasing time $T_{2}=2 /$ $\gamma_{a b}$. The dephasing time is made up of energy relaxing, $T_{1}$, and pure phase disturbing processes, $T_{\mathrm{ph}}$ : $1 / T_{2}=1 / 2 T_{1}+1 / T_{\mathrm{ph}}$. Note, that $T_{\mathrm{ph}}$ and $T_{2}$ are decay constants of amplitudes, while $T_{1}$ corresponds to the decay of energy (population). The spectral width of a homogeneously broadened transition observed in stationary experiments is determined by the dephasing time $T_{2}, \Delta \nu_{\text {spont }}=1 / \pi T_{2}$. Phase disturbing collisions make the spectral lines broader than the natural linewidth $\Delta \nu_{\text {nat }}=1 / 2 \pi T_{1}$.

The spectral resolution may be improved beyond the limit imposed by the spontaneous linewidth $\Delta \nu_{\text {spont }}$ by coherent transient interaction between an electromagnetic field and the atomic system. We introduce an observable quantity, the expectation value of the transition operator $\langle r\rangle=r_{a b}\left(\rho_{a b}+\rho_{b a}\right)$. It has been shown that $\langle r\rangle$ obeys the equation of a damped harmonic oscillator with driving force $\widetilde{A}(t)$ [11]. One obtains from eq. (1) to (3):

$\langle r\rangle+\left(2 / T_{2}\right)\langle r\rangle+\omega_{0}^{2}\langle r\rangle \propto \widetilde{A}(t)$.

Introducing plane waves for $\widetilde{A}(t)$ and $\langle r\rangle$ with slowly varying amplitudes propagating in the $x$-direction we write:

$\widetilde{A}=(1 / 2) A(t) \exp \left(-\mathrm{i} \nu t+\mathrm{i} k_{\mathrm{A}} x\right)+$ c.c.

$\langle r\rangle=(1 / 2) R(t) \exp \left(-\mathrm{i} \omega t+\mathrm{i} k_{\mathrm{R}} x\right)+$ c.c.

and obtain

$\partial R / \partial t+\left[\mathrm{i}\left(\omega_{0}-\omega\right)+1 / T_{2}\right] R=\kappa A(t)$.

$\kappa$ stands for a proportionality constant, $\omega$ is the momentary frequency of the transition amplitude.

During the excitation process we have $\omega=\nu$, i.e. the system is driven off resonance by $\Delta \omega=\omega_{0}-\nu$. Eq. (7) is readily integrated to give

$$
\begin{aligned}
& R(t, \Delta \omega)=\kappa \exp \left(-t / T_{2}\right) \\
& \quad \times \int_{-\infty}^{t} \exp \left[\left(\mathrm{i} \Delta \omega+1 / T_{2}\right) t^{\prime}\right] A\left(t^{\prime}\right) \mathrm{d} t^{\prime} .
\end{aligned}
$$

Depending upon the specific experiment $R(t)$ can be observed directly or may be interrogated by an additional probing light field $E_{\mathrm{p}}(t)$. For a one-photon resonance, $R$ is proportional to a radiating polarisation and $|R|^{2}$ is measured, for instance, in a transmission experiment using a subsequent pulse. For a non-radiative transition (e.g. a Raman process), $R$ may be monitored by coherent scattering of a probe field $E_{\mathrm{p}}$. The scattered field $E_{\mathrm{s}}$ is deduced from Maxwell's equations containing the nonlinear polarisation $P^{\mathrm{NL}} \propto R(t) \times E_{\mathrm{p}}(t)$ as a source term. It has been shown in ref. [12] that the growth of $E_{\mathrm{s}}$ may be written:

$\partial E_{\mathrm{s}} / \partial x \propto R(t) \times E_{\mathrm{p}}(t)$.

\section{Principle of sub-natural linewidth spectroscopy}

Up to this point we have treated the atomic system neglecting the question of the spectral resolution. From text books on quantum mechanics we learn that Heisenberg's uncertainty principle limits the accuracy of measuring spectral features by the relation:

$\Delta E \times \Delta t \geqslant \hbar \quad$ or $\quad \Delta \omega \Delta t \geqslant 1$,

where $\Delta E, \Delta \omega, \Delta t$ denote the inherent uncertainties of the simultaneous measurement of energy ( $\propto$ frequency) and time.

In practical experiments the quantities $\Delta E$ or $\Delta \omega$ are directly observed, e.g., at the image plane of a high resolution spectrographic device.

The time uncertainty $\Delta t$ requires some consideration. It is determined by the experimental observation time and the time constant of the quantum mechanical system. In steady-state experiments $\Delta t$ is determined by intrinsic time constants:

(i) The energy relaxation time $T_{1}$ which is obtained from the observation of the exponential decay of the occupation of the upper quantum state.

(ii) The phase-relaxation time $T_{\mathrm{ph}}$ defined by the phase disturbance of the atomic state, e.g. by collisions. 
Combining the two relaxation rates one obtains the quantum mechanical observation time $\Delta t \approx T_{2}$, where $1 / T_{2}=\left(1 / 2 T_{1}+1 / T_{\mathrm{ph}}\right)$.

Spectral resolution is improved by increasing the observation time beyond $T_{2}$. Two new approaches to the problem using transient coherent experiments are presented in the following sections (4.2 and 4.3).

\section{Spectral resolution of different experiments}

We now wish to discuss the application of eqs. (8) and (9) to several specific experimental situations.

\subsection{Steady-state spectroscopy}

A stationary driving force with amplitude $A_{0}$ and frequency $\nu$ excites the quantum system. One measures the absolute square of the transition amplitude $|R|^{2}$ (e.g. the absorption) at some arbitrary time $T$ tuning the frequency $v$ through the resonance $\omega_{0}$. Eq. (8) yields with $\Delta \omega=\omega_{0}-\nu$

$|R(\Delta \omega)|^{2}=\kappa A_{0}^{2} /\left[\Delta \omega^{2}+\left(1 / T_{2}\right)^{2}\right]$.

As expected, in the steady state we obtain a lorentzian line where the line width (FWHM) is equal to the spontaneous width $\Delta \nu_{\text {spont }}=1 / \pi T_{2}$.

\subsection{Transient excitation using a gaussian shaped pulse}

The quantum system is excited by a pulsed driving force of tunable carrier frequency $\nu$ and of gaussian temporal shape $A(t)=A_{0} \exp \left[-\left(t / t_{\mathrm{p}}\right)^{2} 2 \ln 2\right]$. As shown below the pulse duration $t_{\mathrm{p}}$ should be longer than the dephasing time $T_{2}$ of the excited system. Using eq. (8) we obtain

$$
\begin{aligned}
& |R(T, \Delta \omega)|^{2}=\kappa A_{0}^{2} \exp \left[-2 T / T_{2}-\left(t_{\mathrm{e}} / t_{\mathrm{p}}\right)^{2} 4 \ln 2\right] \\
& \quad \times\left|\int_{-\infty}^{T} \exp \left(\mathrm{i} \Delta \omega t^{\prime}\right) \exp \left[-\left|\left(t^{\prime}-t_{\mathrm{e}}\right) / t_{\mathrm{p}}\right|^{2} 2 \ln 2\right] \mathrm{d} t^{\prime}\right|^{2}
\end{aligned}
$$

with $t_{\mathrm{e}}=t_{\mathrm{p}}^{2} /\left(T_{2} 4 \ln 2\right)$. The time $T$ corresponds to the time of observation. For late times $T$ the integral of eq. (12) is proportional to the Fourier transform of the gaussian driving pulse; i.e., the frequency dependence of $|R|^{2}$ has the same gaussian shape as the driving force. Or, in other words, the observed frequency bandwidth is determined by the pulse dura- tion $t_{\mathrm{p}}$ and not by the dephasing time $T_{2}$ (as in the preceding section). Experimentally one observes $|R|^{2}$ as a function of the frequency detuning $\Delta \omega$ of the driving pulse and finds a bandwidth $\Delta \nu_{\text {coh }}$ at a delayed time $T$.

We introduce the ratio $V=\Delta \nu_{\text {spont }} / \Delta \nu_{\text {coh }}$ which defines the spectral narrowing found in the transient experiment discussed here. For late times $T, \Delta v_{\mathrm{coh}}$ is determined by the gaussian pulse of duration $t_{\mathrm{p}}$ and one obtains $V=\Delta \nu_{\text {spont }} / \Delta \nu_{\text {Gauss }}=t_{\mathrm{p}} / 1.4 T_{2} ;$ according to this relation one observes a line narrower than the spontaneous one for $t_{\mathrm{p}}>1.4 T_{2}$.

The question now arises concerning the magnitude of $|R|^{2}$ at a late observation time $T$. In fig. 1 a the ratio of the observed linewidth $\Delta \nu_{\text {coh }}$ to the spectral width of the gaussian pulse $\Delta \nu_{\text {coh }} / \Delta \nu_{\text {Gauss }}$ is plotted versus $T / t_{\mathrm{p}}$ for different values $\Delta \nu_{\text {spont }} / \Delta \nu_{\text {Gauss }}$ (i.e. for different $T_{2}$ ). As seen from fig. la, $\Delta \nu_{\mathrm{coh}} / \Delta \nu_{\mathrm{G}}$ auss
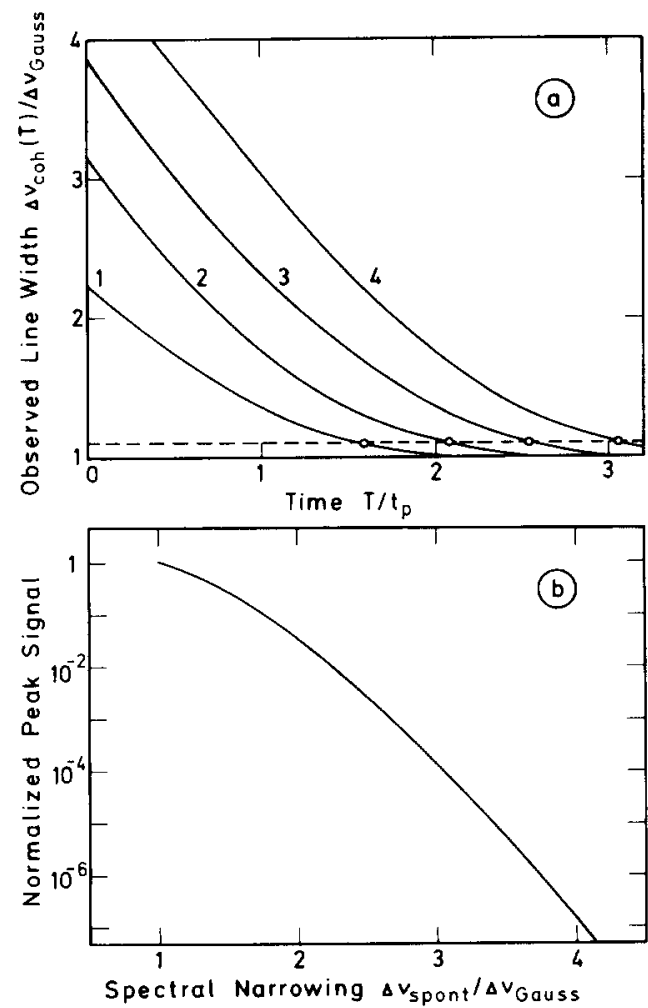

Fig. 1. (a) Narrowing of observed linewidth versus time of delayed observation. The exciting gaussian pulse has a wid th $\Delta \nu_{\text {Gauss }}$ and a duration $t_{\mathrm{p}}$. The parameter is given by $\Delta v_{\text {spont }} / \Delta v_{\text {Gauss }}$ where $\Delta v_{\text {spont }}$ is the spontaneous (steady-state) linewidth. (b) Normalized peak of signal (transition amplitude squared $\left.|R(T)|^{2}\right)$ versus spectral narrowing. 
approaches unity for sufficiently large $T / t_{\mathrm{p}}$ values. Larger line narrowing $V$ requires later observation times $T$. The points on the broken line mark the $T / t_{\mathrm{p}}$ positions where the observed linewidth $\Delta \nu_{\text {coh }}$ is $10 \%$ longer than the linewidth $\Delta \nu_{\text {Gauss }}$ of the applied gaussain pulse. As an example we discuss the case of $V=4$ and $T / t_{\mathrm{p}}=3$. With pulses of $t_{\mathrm{p}} \geqslant 5.6 T_{2}$ and for a delayed observation time of $T=16.8 T_{2}$ one obtains a line narrowing by a factor of 3.6 .

According to eq. (12) the signal $|R(T, \Delta \omega)|^{2}$ decreases strongly for later observation times $T$. In fig. $1 \mathrm{~b}$ the peak signal intensity at $\Delta \omega=0$ is plotted versus the spectral narrowing $\Delta \nu_{\text {spont }} / \Delta \nu_{\text {Gauss }}$ for a constant dephasing time $T_{2}$ and for a constant amplitude $A_{0}$ of the driving force. Calculations were made for $\Delta \nu_{\text {coh }} / \Delta \nu_{\text {Gauas }}=1.1$ (see broken line in fig. 1a). Larger spectral narrowing gives rise to strongly reduced signal intensities. In practical cases, the optimum spectral resolution is determined by the signal to noise ratio of the experimental system.

The accurate determination of the absolute value of the transition frequency $\omega_{0}$ requires both, a narrow spectral response of the excited system and a high precision of the tunable excitation frequency $\nu$. The accuracy of the latter depends upon the band width of the exciting pulse. Gaussian shaped excitation pulses allow to determine the system response and the excitation frequency $\nu$ with equal precision proportional to $\Delta \nu_{\text {Gauss }}$. Exponentially decaying pulses have a lorentzian Fourier transform (in ref. [8] equal to the spontaneous line width) making the determination of $\nu$ less accurate.

\subsection{Short excitation and prolonged interrogation (SEPI) spectroscopy}

Very recently, we have demonstrated a technique to obtain subline width resolution of Raman transitions $[9,10]$. The method is shown schematically in fig. 2. A short driving force $A(t)$ at a frequency $\nu$ near the resonance $\omega_{0}$ of the quantum system generates a transition amplitude $R(t)$ (see fig. 2a). The driving force has a short duration or is switched off rapidly at $t=0$. After the excitation the exponentially decaying transition amplitude $R(t)$, oscillating freely at $\omega_{0}$, is investigated by a prolonged probing pulse at frequency $\omega_{\mathrm{p}}$. A pulse with suitably shaped amplitude $E_{\mathrm{p}}(t)$ generates the scattered signal $E_{\mathrm{s}}(t)$ (see eq. (9))

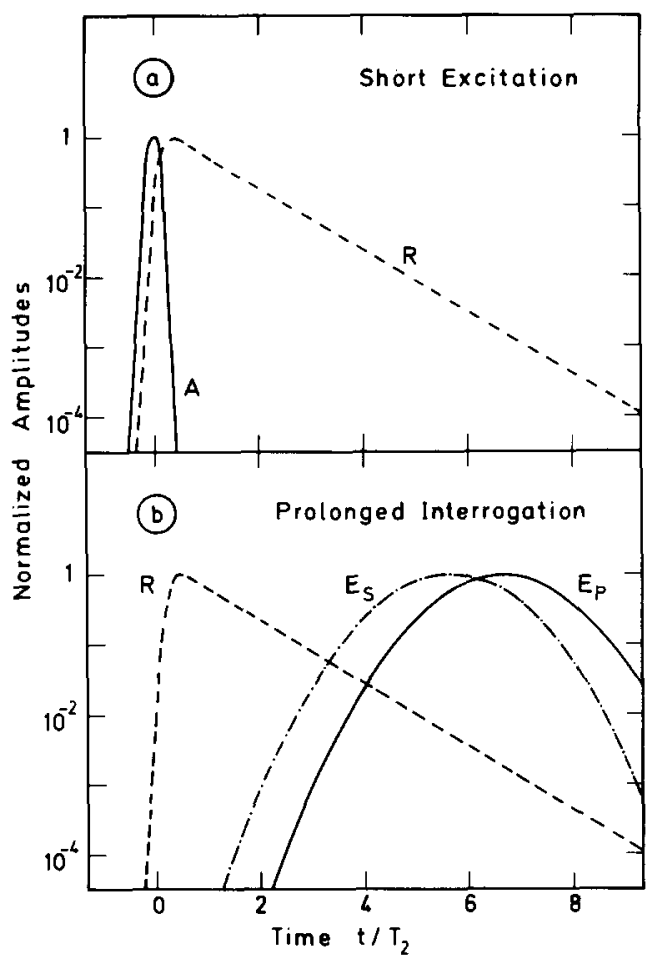

Fig. 2. The short excitation and prolonged interrogation (SEPI) technique. (a) A short driving pulse, $A(t)$, excites the exponentially decaying transition amplitude $R(t)$. (b) The transition amplitude $R(t)$ is interrrogated by a long pulse $E_{\mathrm{p}}$ giving rise to the scattered pulse $E_{\mathrm{S}}(t)$.

with frequency $\omega_{\mathrm{s}}=\omega_{\mathrm{p}} \pm \omega$. The frequency dependence of the scattered intensity $I_{\mathrm{s}}\left(\omega_{\mathrm{s}}\right) \propto\left|E_{\mathrm{s}}\left(\omega_{\mathrm{s}}\right)\right|^{2}$ has the form

$$
\begin{aligned}
& I_{\mathrm{s}}\left(\omega_{\mathrm{s}}\right) \propto\left|\int_{-\infty}^{+\infty} \mathrm{d} t \exp \left(\mathrm{i} \omega_{\mathrm{s}} t\right) E_{\mathrm{s}}(t)\right|^{2} \\
& \propto\left|\int_{-\infty}^{+\infty} \exp \left(\mathrm{i} \omega_{\mathrm{s}} t\right) E_{\mathrm{p}}(t) R(t) \exp \left[-\mathrm{i}\left(\omega_{\mathrm{p}} \pm \omega\right) t\right] \mathrm{d} t\right|^{2} .
\end{aligned}
$$

Introducing $\Delta \omega=\omega_{\mathrm{s}}-\omega_{\mathrm{p}} \pm \omega_{0}$ and $t_{\mathrm{e}}=t_{\mathrm{p}}^{2} /\left(T_{2} \times\right.$ $4 \ln 2)$ and using a gaussian shape $E_{\mathrm{p}}(t)=E_{\mathrm{p} 0}$ $\exp \left[-\left(\left(t-T_{\mathrm{D}}\right) / t_{\mathrm{p}}\right)^{2} 2 \ln 2\right]$ for the delayed probing pulse we obtain at late delay time $T_{\mathrm{D}}$ a gaussian shaped spectrum centered at the frequency $\omega_{p} \pm \omega_{0}$ :

$$
\begin{aligned}
& I_{\mathrm{s}}\left(T_{\mathrm{D}}, \Delta \omega\right) \propto \exp \left[-2 T_{\mathrm{D}} / T_{2}-\left(t_{\mathrm{e}} / t_{\mathrm{p}}\right)^{2} 4 \ln 2\right] \\
& \quad \times\left|\int_{-T_{\mathrm{D}}}^{\infty} \exp (\mathrm{i} \Delta \omega t) \exp \left[-\left(t-t_{\mathrm{e}} / t_{\mathrm{p}}\right)^{2} 2 \ln 2\right] \mathrm{d} t\right|^{2}
\end{aligned}
$$


For a long delay time $T_{\mathrm{D}}$ the width of the observed spectrum is only determined by the duration of the probing pulse. For a sufficiently long pulse, $t_{\mathrm{p}}>1.4$ $T_{2}$, the SEPI lines are narrower than the spontaneous width.

The favorable features of the SEPI spectroscopy have been confirmed in Raman type experiments of homogeneously broadened vibrations in liquids $[9,10]$.

We briefly point to another promising application of the SEPI technique, the study of atomic twophoton transitions. Two counter-propagating short pulse of frequencies $\omega_{1}=\omega_{2}$, close to $\omega_{0} / 2$, coherently excite the quantum system. The transition amplitude $R(t)$ subsequently decays exponentially. The excited volume should be monitored by a third delayed long pulse of gaussian shape with center frequency $\omega_{3} \approx \omega_{0} / 2$. A signal pulse of frequency $\omega_{4}=$ $\omega_{0}-\omega_{3}$ is generated in opposite direction to the pulse of frequency $\omega_{3}$ and may be analyzed in a spectrometer. The suggested geometry eliminates Doppler broadening and the SEPI experiment reduces the homogeneous line width.

\section{Summary and conclusions}

The salient features of the two techniques discussed in the preceding section are as follows:

4.2. A long gaussian shaped driving force $A(t)$ excites the two-level system (pulse duration $t_{\mathrm{p}}$ longer than the dephasing time $T_{2}$ ). We wait for some time $T>t_{\mathrm{p}}$ and observe the coherent transition amplitude $R .|R|^{2}$ may be monitored by a gated photodetector or by a transmission experiment using a second short pulse. Varying the frequency of the exciting pulse one obtains an absorption band which is narrower than the one known from spontaneous steady-state measuremntes. This technique requires exciting pulses of appropriate shape with frequency tunability over the resonance of interest. It is applicable to electronic transitions in the UV, the visible and the near IR where dye lasers are available.
4.3. (SEPI): A short pulse excites the quantum mechanical system $\left(t_{\mathrm{p} 1} \leqslant T_{2}\right)$. At some time $T$ after the excitation $\left(T>t_{\mathrm{p} 1}\right)$ a long gaussian shaped pulse coherently probes the transition amplitude $R(T)$ generating a scattered signal. The narrow spectrum of the scattered signal is observed. As a result, short pulses for excitation and long pulses for probing are needed. The technique is particularly useful when direct resonant excitation is not possible, e.g. for Raman or two-photon transitions.

Both methods provide improved spectral resolution. Gaussian pulses are treated for two reasons: (i) Temporally long gaussian in put pulses produce sharp gaussian shaped spectra without disturbing ringing and (ii) gaussian shaped pulses are readily realized in a number of laser systems, e.g. mode-locked Nd-lasers. Calculations and experiments show that under practical conditions the spectral width may be reduced by a factor of four below the spontaneous linewidth.

\section{References}

[1] For a review see: $H$. Walther, in: Laser spectroscopy of atoms and molecules, Topics in Applied Physics, Vol. 2, ed. H. Walther (Springer, Heidelberg, 1976) p. 1

[2] G. Copley, B.P. Kibble and G.W. Series, J. Phys. B1 (1968) 724

[3] H. Figger and H. Walther, Z. Physik 267 (1974) 1.

[4] H. Metcalf and W. Phillips, Optics Lett. 5 (1980) 540.

[5] F. Shimizu, K. Umezu and H. Takuma, Phys. Rev. Lett. 47 (1981) 825.

[6] P. Meystre, M.O. Scully and H. Walther, Optics Comm. 33 (1980) 153.

[7] H.-W. Lee, P. Meystre and M.O. Scully, Phys. Rev. A24 (1981) 1914.

[8] P.E. Coleman, D. Kagan and P.L. Knight, Optics Comm. 36 (1981) 127

[9] W. Zinth, Optics Comm. 34 (1980) 479

[10] W. Zinth, M.C. Nuss and W. Kaiser, Chem. Phys. Lett. 88 (1982) 257

[11] J.A. Giordmaine and W. Kaiser, Phys. Rev, 144 (1966) 676.

[12] R.L. Carman, F. Shimizu, C.S. Wang and N. Bloembergen, Phys. Rev. A2 (1970) 60; A. Laubereau and W. Kaiser, Rev. Mod. Phys. 50 (1978) 607. 